\title{
Simulation of Fluidized Beds and Other Fluid- Particle Systems Using Statistical Mechanics
}

\author{
Kevin D. Seibert and Mark A. Burns \\ Dept. of Chemical Engineering, University of Michigan, Ann Arbor, MI 48109
}

\begin{abstract}
The expansion behavior and structural phenomena of fluid-particle systems was simulated using a method analogous to the Monte Carlo method for molecular systems. Individual particles are moved, and the resulting moves are accepted or declined based on the change in the system's potential energy and the average kinetic energy of the system. Several fluid-particle systems have been successfully predicted with the model including colloidal particle concentration profiles and random packing of uniform spheres. Additionally, predictions of steady-state fluidized-bed expansion characteristics for uniformly sized stainless-steel spheres and narrowly distributed nickel and glass spheres show excellent agreement with the theoretical model used in the simulation and satisfactory agreement with experimental data. Dynamic expansion predictions of both bed height and overall bed structure as a function of time also agree with the experimental data.
\end{abstract}

\section{Introduction}

Fluidized beds are used in a variety of applications, such as catalytic reactors and expanded bed adsorbers. Knowledge of the expansion characteristics of fluidized beds is crucial in predicting the rate of mass transfer and the contact time between phases in the bed. For this reason, the velocity/bedheight or velocity/voidage relationship for the expansion of uniformly sized particles as well as mixtures of particle sizes and densities has been studied extensively.

Many models exist for predicting the behavior and structure of fluidized beds. Several theoretical models (Brinkman, 1947; Happel, 1958; Kuwabara, 1959) have been proposed, with applicability at low particle Reynolds numbers. Most of the semitheoretical (Steinour, 1944; Loeffler and Ruth, 1959; Oliver, 1961; Letan, 1974; Foscolo et al., 1983; Jean and Fan, 1989) and empirical (Lewis et al., 1949; Jottrand, 1952; Lewis and Bowerman, 1952; Richardson and Zaki, 1954a; Wen and Yu, 1966; Barnea and Mizrahi, 1973; Wen and Fan, 1974; Garside and Al-Dibouni, 1977; Rapagna et al., 1989; Hirata and Bulos, 1990) correlations that have been suggested to describe the steady-state expansion characteristics of fluidized beds establish a relationship between the superficial fluid velocity and the expanded bed voidage. By far the most widely accepted form for correlations is the Richardson-Zaki relationship (Richardson and Zaki, 1954b), which relates superficial fluid velocity to terminal velocity as a function of the bed voidage.
We have chosen to model the behavior and spatial phenomena of fluidized and stationary particle systems from a fundamental standpoint using techniques analogous to statistical mechanical molecular simulations. Molecular simulations can be used to predict a wide variety of system properties, including thermodynamic properties and molecular arrangements (Gubbins and Panagiotopoulos, 1989; Ciccotti et al., 1987), but have yet to be widely used to predict the properties of fluid-particle systems. Many fluid-particle systems are ideally suited to be simulated using statistical mechanical methods because they rely on the interactions of the particles with other particles and with the surrounding fluid.

Recent work on the use of statistical mechanical techniques to simulate fluid-particle systems has been summarized by Dickinson and Euston (1992). Early simulations were used to investigate static colloidal systems and were focused on aggregation and flocculation of colloidal particles (Snook and van Megen, 1975; Hirtzel and Rajagopolan, 1985; Dickinson, 1990; Vold, 1963; Hutchinson and Sutherland, 1965; Sutherland, 1966) and on the adsorption of polymeric molecules in colloidal suspensions (Vincent and Whittington, 1982; Takahashi and Kawaguchi, 1982; Fleer and Lyklema, 1983; Fleer et al., 1988). To date, few studies have been published on the simulation of the structure of colloidal systems or the structure of other fluid-particle systems using statistical mechanical techniques. 
In our simulation, we use the analysis of the forces that affect particle movement to determine the structural and macroscopic properties of several fluid-particle systems. In addition to being purely predictive, this method has the advantage of allowing the visualization of microscopic structural phenomena that occur in fluid-particle systems by analyzing the position of individual particles. The primary disadvantage to this method is the computationally intensive nature of the simulations.

Although a variety of techniques have been used in statistical mechanical simulations, most of the techniques can be classified into two general categories: molecular dynamic (MD) or Monte Carlo (MC) simulations. In MD simulations, molecular movement is based upon the overall system energy and interactions with neighboring molecules. An analogous particle simulation would include, among other things, the transfer of momentum between particles and the calculation of viscous forces based on the fluid flow field. This approach to particle simulations would allow for the accurate prediction of dynamic behavior of a particle system, but the computational intensity of the calculations would far exceed any practical limits on even the fastest computers currently available.

An alternative approach to momentum conservation algorithms for particle simulations is a method analogous to the MC method for molecular simulations. MC simulations use random number generators to move molecules based on acceptance probabilities. The basis for molecular movement is the kinetic energy of the system that accounts for the random fluctuations of molecules. The advantages of MC simulations over MD simulations are the ease of programming and shorter computation times. In the analogous MC simulation for particle systems, the system is driven toward a steady-state condition by the external hydrodynamic, buoyant, and gravity forces affecting the energy change of the system.

In this article, we show the development of a computer simulation and its ability to predict the macroscopic properties of several particle systems. Due to the fundamental nature of the model, we have used this single model to predict the concentration profiles in colloidal suspensions, the random packing of spherical particles, and the static and dynamic expansion characteristics of fluidized beds.

\section{Model Description}

The macroscopic particle systems are modeled using a small, representative number of particles. The simulation space consists of a solid bottom, infinite height in the $Y$ direction, and fully periodic boundaries in the $X$ and $Z$ directions (Figure 1). Note that the periodic boundaries in the $Y-Z$ and $Y-X$ planes allow for an essentially infinite system. By designing the simulation volume in this way, it is possible to choose a representative system large enough to remove any repeating boundary effects from the periodic boundaries or edge effects from the solid surface at the bottom of the simulation space.

\section{Random placement and motion}

A typical simulation is performed by randomly placing 500-5,000 particles within the simulation boundaries. A

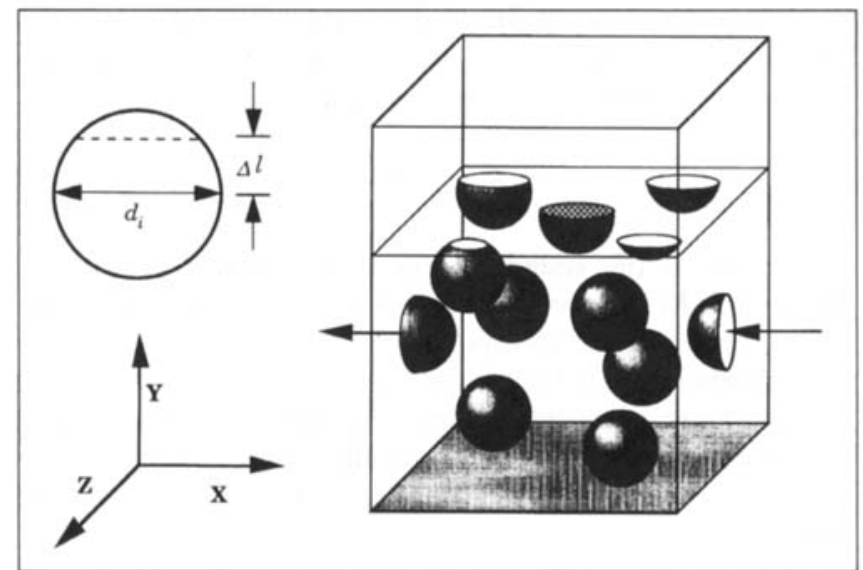

Figure 1. Example of simulation space.

The simulation space has periodic boundaries in the $X$ and $Z$ direction, a solid bottom, and infinite height in $Y$ direction. Local void fraction is calculated by passing a plane through the center of the selected particle (crosshatched) and computing the contribution to the intersected plane area from each intersected particle. The tops of the particles have been removed to show intersected area.

pseudorandom number generator computes random particle coordinates, and particles are sequentially placed in the simulation space in a nonoverlapping configuration. If an attempted particle placement results in an overlap with another particle, the particle is removed and another set of center coordinates is generated until all particles have been placed in the simulation space. Although the simulation space available for particle movement has infinite height in the $Y$ direction, particles are initially placed in a space equivalent to three times the size of the repeating boundary in the $X$ and $Z$ directions. With this type of particle placement, a simulation space containing 10 particles per repeating bed width can easily accommodate a system of 1,000 particles on initial placement. For larger systems, or fewer particles in a repeating bed width, the initial placement height is modified to accommodate particle placement.

Once particles have been placed in the simulation space, particle movement begins with the random selection of a single particle in the representative system. The selected particle is moved a fixed distance $S$ in a random direction in three dimensions. A particle moving a fixed step size therefore has all the positions in a shell of radius $S$ available for movement. To ensure equal probability of all positions rather than all angles in a spherical coordinate system, $\phi$ and $\cos \theta$ are randomly chosen, and converted to their RCCS counterparts. If the movement of the particle results in an overlap with an adjacent particle, the selected move is automatically rejected and the particle is returned to the original coordinates. A nonoverlapping move is accepted or declined based upon the acceptance criteria.

\section{Acceptance criteria}

Nonoverlapping particle moves are accepted based upon the change in system energy generated by the move. Energetically "favorable" moves (moves that result in a lowering of the system energy) are accepted with $100 \%$ probability. An energetically "unfavorable" move may still be accepted, but only with some finite probability. 
Analogous to the Monte Carlo method for molecular simulations, the acceptance probability $\left(p_{a}\right)$ of an energetically unfavorable move is given by the equation

$$
p_{a}=e^{-\frac{\Delta E}{K E}}
$$

where $\Delta E$ is the system energy change resulting from the move and $K E$ is the average kinetic energy of the system. Once the change in the system's energy is calculated, a random probability $\left(p_{r}\right)$ between 0 and 1 is generated and the particle move is accepted if $p_{a}$ is greater than this value. A declined move due to a failure of the acceptance criteria resets the selected particle's coordinates to their previous values.

\section{Energy calculations}

In our simulation, the system energy change, $\Delta E$, is the change in potential energy due to particle movement and is calculated using gravity, buoyancy, and fluid dynamic forces. Although we have ignored particle-particle forces such as electrostatic, frictional, or magnetic forces, the form of the simulation allows these interactions to be easily incorporated into the energy calculation. The combined gravity and buoyancy force affecting a submerged body (i.e., all the forces except the fluid forces) is given by

$$
F_{g, b}=\left(\rho_{p}-\rho_{f}\right) V_{p} g
$$

where $F$ is the force on a particle, $\rho_{p}$ is the particle density, $\rho_{f}$ is the fluid density, $V_{p}$ is the particle volume, and $g$ is the acceleration due to gravity.

Several mathematical models (Brinkman, 1947; Happel, 1958; Kuwabara, 1959) have been derived for the fluid forces acting on a particle in a flow field. For low Reynolds number flows, the microscopic fluid dynamic force influencing a particle is well represented by Happel's solution to the equation of motion around random assemblages of spheres. This force is given by

$$
F_{f}=\left(\frac{3+2 \gamma^{5}}{2-3 \gamma+3 \gamma^{5}-2 \gamma^{6}}\right) 4 \pi \mu d_{p} U_{s}
$$

where

$$
\gamma=(1-\epsilon)^{1 / 3},
$$

$\mu$ is the fluid viscosity, $d_{p}$ is the particle diameter, and $\epsilon$ is the local void fraction surrounding a particle. For high Reynolds number flows, we use the Ergun equation:

$$
\frac{\Delta p}{L}=\frac{150 \mu U_{s}}{d_{p}^{3}} \frac{(1-\epsilon)^{2}}{\epsilon^{3}}+\frac{1.75 p_{f} U_{s}^{2}}{d_{p}} \frac{(1-\epsilon)}{\epsilon^{3}},
$$

where the hydrodynamic force, $F_{f}$, is given by

$$
F_{f}=\frac{\Delta p}{L} \frac{V_{p}}{(1-\epsilon)} .
$$

The fluid force calculated using either of these equations is a strong function of $\epsilon$, the local void fraction. If the particles are uniformly distributed in a given space, this local value is identical to the average void fraction of the system, $\epsilon_{\mathrm{sys}}$. This void fraction is given by

$$
\epsilon_{\mathrm{sys}}=1-\frac{\left(1-\epsilon_{0}\right) H_{0}}{H},
$$

where $\epsilon_{0}$ is the packed void fraction of the system, $H_{0}$ is the height of the particles when packed, and $H$ is the current or expanded height of the system (same initial width).

For real particle systems, void fractions can be significantly different than the average system void fraction. Davis and Carter (1990) have suggested that the local void fraction surrounding a particle may be calculated by using either a finite subvolume containing the particle, a plane passed through the particle, or a line intersecting the particle. Our simulation uses a horizontal plane intersecting the center of the selected particle and computes the local void fraction from the intersected particle area divided by the total area (Figure 1). The intersected area for each particle, $A_{i}$, is

$$
A_{i}=\pi\left(\frac{d^{2}}{4}-\Delta l^{2}\right)
$$

where $d_{p}$ is the diameter of the selected particle and $\Delta l$ is the distance between the selected particle's center point and the plane of intersection. The local void fraction is then defined as

$$
\epsilon=1-\frac{\sum A_{i}}{A_{t}} \quad \text { where } i=1,2,3, \ldots N,
$$

$A_{t}$ is the unit bed cross-sectional area, and $N$ is the number of intersected particles.

Using Eqs. 2-6, 8, and 9, the net change in system energy, $\Delta E$, resulting from an individual particle move can then be calculated from the product of the force on the particle $\left(F_{g, b}\right.$ $+F_{f}$ ) and the vertical distance traveled by the particle $\left(Y_{\text {old }}-Y_{\text {new }}\right)$. This technique for calculating the change in system energy, which assumes that the forces remain constant over the entire move distance, is valid for small step sizes.

The change in system energy is then substituted into Eq. 1 and the result compared to $p_{r}$ to determine if the specific move is accepted or declined. Once such a single particle move has been completed, the process (selection, movement, overlap check, and acceptance check) is repeated until the system converges to a steady-state configuration. Steady state is determined by an absence in change in the overall system void fraction and any macroscopic structural arrangements that may arise in the particle bed as a function of simulation time.

\section{Experimental Studies}

Materials and equipment

Liquid fluidization runs were performed using $25^{\circ} \mathrm{C}$ distilled/deionized water and commercial-grade mineral oil as 
the fluidizing medium. Nitrogen at $25^{\circ} \mathrm{C}$ was used for all gas fluidized beds. Expansion data was taken in $1.5-$ and $2.5-\mathrm{cm}$ ID Kontes Flex-Column chromatography columns. The columns were fitted with a porous distributor $0.32 \mathrm{~cm}$ thick, with pore sizes ranging from 25 to $50 \mu \mathrm{m}$. The fluid was pumped using a model QD laboratory pump from Fluid Metering, Inc. for liquid fluidization data. Expansion data were obtained by fluidizing stainless-steel spheres $\left(d_{p}=0.1588 \pm\right.$ $0.0003 \mathrm{~cm}, \rho=7.63 \mathrm{~g} / \mathrm{cm}^{3}$ ) from Winsted Precision Ball Company, nickel spheres $\left(\bar{d}_{p}=150 \mu \mathrm{m}, \rho=8.47 \mathrm{~g} / \mathrm{cm}^{3}\right)$ from Johnson Matthey, and glass beads (high index, $\bar{d}_{p}=134 \mu \mathrm{m}$, $\rho=4.42 \mathrm{~g} / \mathrm{cm}^{3}$; low index, $\bar{d}_{p}=240 \mu \mathrm{m}, \rho=2.45 \mathrm{~g} / \mathrm{cm}^{3}$ ) from Potters Industries. The simulation program used for configuration generation, spatial resolution, and position analysis was written in FORTRAN and Absoft's MacFortran II for the Macintosh. Computer trials were run on IBM RS6000 and SUN Sparcstation computers. Particle distribution information was calculated from projected area measurements with Biological Detection Systems-Image Version 1.2 image analysis software, a Macintosh IIfx computer, a Hammamatsu camera, and a Zeiss Axioscop microscope.

\section{Methods}

Velocity -Voidage Data. Velocity-voidage data were taken by adding a known, dry mass of particles to a graduated chromatography column and allowing the particles to settle. Initial bed depths ranged from 8 to $15 \mathrm{~cm}$. The fluid was pumped up through a porous distributor at the bottom of the column and through the bed of particles. When operating the gas fluidized bed, the gas was directed through the porous distributor and particle bed and finally through a flowmeter. Void fractions were calculated using Eq. 7.

Particle Data. The average particle diameter was determined using 600 particles from each particle type. Particles were randomly chosen and the average diameters were calculated using the Sauter mean particle diameter of the distribution.

Particle density was calculated by weighing a volume of particles in a volumetric flask. Water at $25^{\circ} \mathrm{C}$ was added to the flask, and the flask was shaken to remove any air bubbles. The density of the particles is then calculated from the known mass of particles and the difference in the flask volume and the known volume of water present (calculated from the mass of the water added to the flask and the density of the water at that temperature).

\section{Results and Discussion}

\section{Model precision}

The final steady-state configurations of simulated particle systems must be independent of the simulation parameters. We can use a model particle system, such as a fluidized bed, to determine the effect of step size, particles per repeating bed width, initial bed depth, and initial bed configuration on the steady-state particle arrangement. For each of these parameters, values should be chosen that facilitate a rapid approach to steady state, but do not affect the final steady-state structure.

The step size (random movement distance), $S$, is one parameter that can slow the approach to the steady-state con-

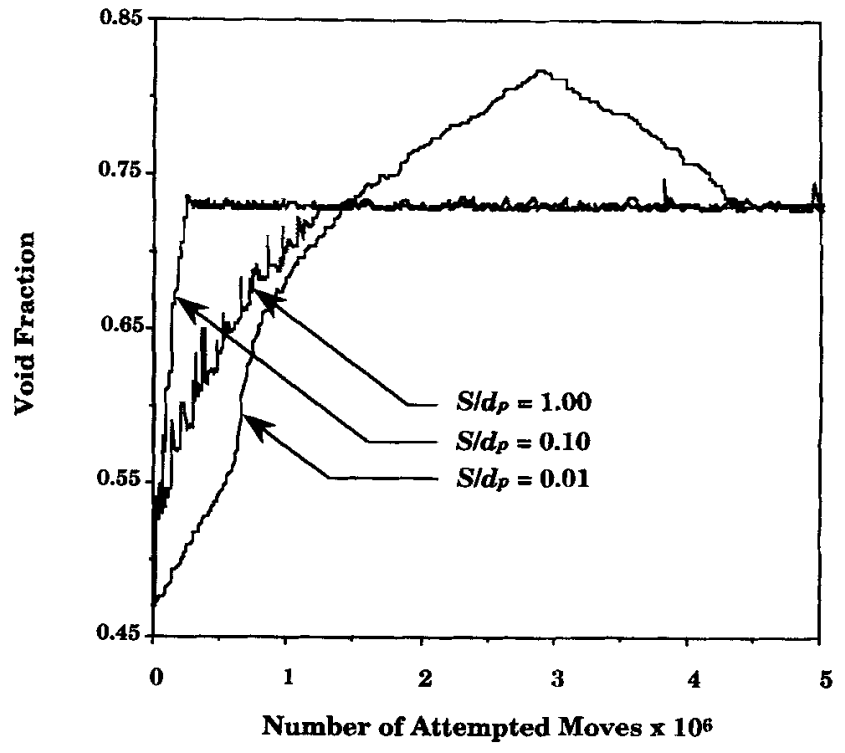

Figure 2. Effect of step size on approach to steady state.

By simulating the expansion of glass particles $\left(d_{p}=134 \mu \mathrm{m}\right.$, $\rho_{p}=4.42 \mathrm{~g} / \mathrm{cm}$ ) resulting from a step change in velocity (from $0.05 \mathrm{~cm} / \mathrm{s}$ to $0.5 \mathrm{~cm} / \mathrm{s}$ ), we find that operating at an $S / d_{p}$ ratio of 0.1 results in a rapid approach to steady state and very little noise in the data.

figuration if not chosen properly. As shown in Figure 2, too large a step size results in frequent particle overlapping and slows the system's approach to steady state. Choosing too small a step size increases the time required to reach the steady-state configuration because many more steps are necessary. Ideally, the step size should correspond to the average distance between adjacent particles. This distance varies from $0.06 d_{p}$ at an $\epsilon=0.5$ (10\% expansion), to $0.2 d_{p}$ at an $\epsilon=0.7$ (100\% expansion). Typically, we operate at an intermediate step-size-to-diameter ratio $\left(S / d_{p}\right)$ of 0.1 .

The size of the simulation space can also affect the approach to steady state (more particles require more computation time) and can adversely affect the steady-state configuration. Operating with too small a bed width (defined as the number of particle diameters in a single nonrepeating bed unit) can result in a misrepresentation of the steady-state configuration; too large a bed width results in excessive run times. Figure 3 shows that greater than six particles per unit bed width results in sufficient elimination of the periodicity resulting from the repeating boundaries. By expanding a bed $65 \%$, and comparing the resulting bed height for several initial packed bed depths (Figure 4), we have found that a bed five particles deep (when packed) minimizes the effects from the solid boundary on the bottom of the simulation box. For our simulations we operate with 1,000 particles in the simulation space (10 particle diameters in the $X$ and $Z$ directions, 9.5 particles deep when packed) to eliminate repeating and solid boundary effects.

While the final steady-state configuration may be affected by the dimensions of the simulation space, it is independent of the direction of approach to the final steady state. Figure 5 shows that the void fraction of an expanded bed will be the same regardless of whether the approach initiates from a less expanded state (expansion) or a more expanded state (settling). Note that although the steady-state configurations are 


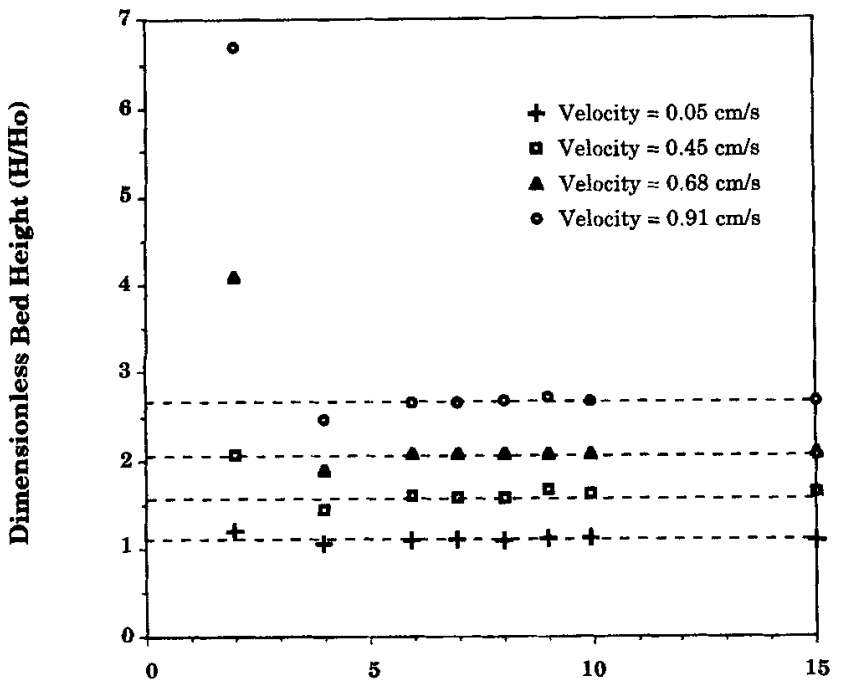

Number of Particles/Screen Width

Figure 3. Simulated bed expansion as a function of bed width.

A bed 15 particles ( $240 \mu \mathrm{m}$ glass, $\rho=2.45$ ) deep, shows no effects from the wall's periodicity beyond 6 particles per screen width.

identical, the number of moves necessary to obtain steady state is very different.

\section{Colloidal and packed particles}

Although a number of fluid-particle systems can be simulated using the technique described in this article, the most obvious system is a colloidal suspension. The motion of colloidal particles is physically and mathematically similar to the motion of molecules in a static fluid. Thermal fluctuations

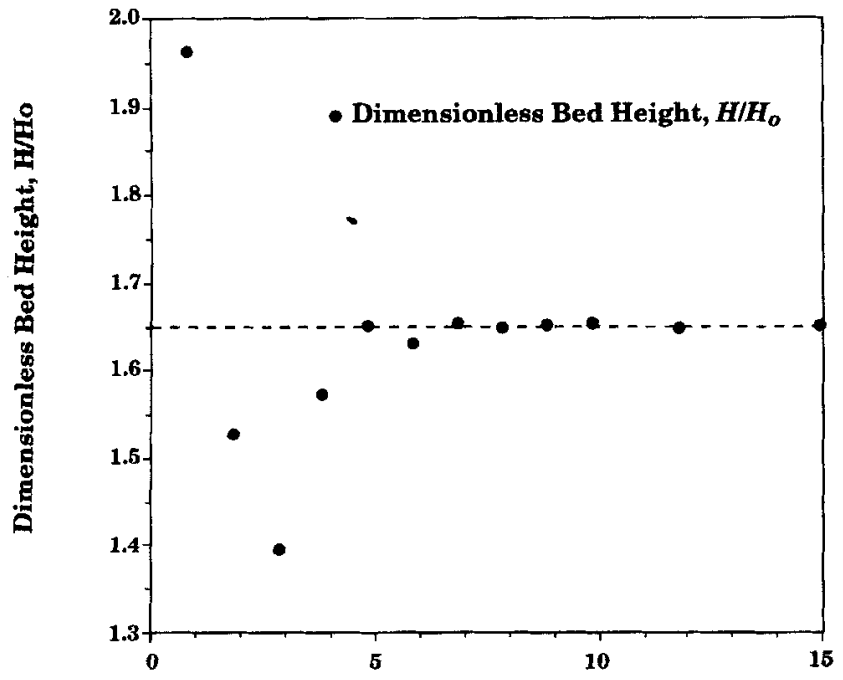

Bed Depth (Particles)

Figure 4. Simulated expansion of 240- $\mu \mathrm{m}$ glass particles as a function of bed depth.

Results indicate that a simulated bed needs to be deeper than approximately 5 particles (when packed) to eliminate effects from the solid surface at the bottom of the simulation box.

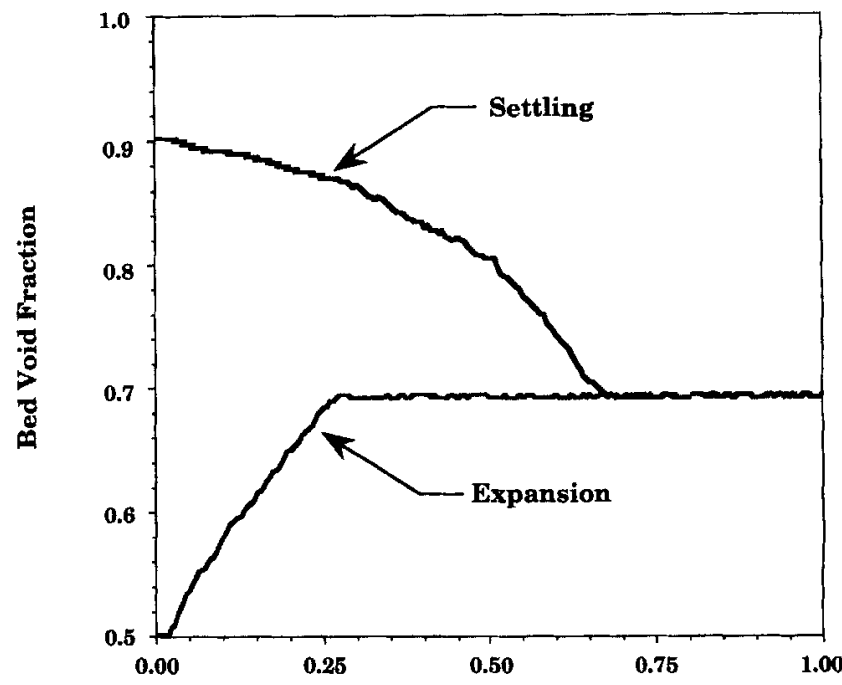

Number of Attempted Moves $\times 10^{6}$

Figure 5. Effect of the direction of approach.

Expansion of a packed bed or settling of randomly placed particles to the same velocity $(0.4 \mathrm{~cm} / \mathrm{s})$ results in the same steady-state void fraction. The particles were 150 -mm-dia glass beads $\left(e=4.5 \mathrm{~g} / \mathrm{cm}^{3}\right)$.

are responsible for the particles' Brownian motion and, therefore, the kinetic energy (Eq. 1) can be defined the same way for this system as it is for molecular systems: the product of the Boltzmann's constant and the average system temperature.

Our simulations of colloidal particles are able to predict the properties of colloidal suspensions. For instance, colloidal particles will form vertical concentration gradients due to the fact that the particles' settling velocity is on the same order as their diffusive flux. Figure 6 shows the steady-state concentration of colloidal particles as a function of distance above the bottom of the simulation space. As expected, the downward settling of the particles is matched by an upward concentration-driven diffusive flux.

The concentration profile generated by our simulations can also be predicted analytically. The expression for the concentration profile is obtained from matching the sedimentation and diffusion fluxes (Hiemenz, 1986):

$$
\frac{d c}{d x}=\frac{m_{p}}{k T}\left(1-\frac{\rho_{f}}{\rho_{s}}\right) g c,
$$

where $m_{p}$ is the mass of a particle, $k$ is Boltzmann's constant, $T$ is the system operating temperature, $\rho_{p}$ is the density of the colloidal particle, $\rho_{f}$ is the density of the surrounding fluid, and $c$ is the concentration at any point, $x$, in the container. By integrating Eq. 10, we obtain the expression for the steady-state concentration profile:

$$
\ln \left(\frac{c_{2}}{c_{1}}\right)=\frac{m_{p}}{k T}\left(1-\frac{\rho_{f}}{\rho_{s}}\right) g\left(x_{2}-x_{1}\right)
$$

where $c_{1}$ and $c_{2}$ are the concentrations in the colloidal suspension at points $x_{1}$ and $x_{2}$, respectively. 


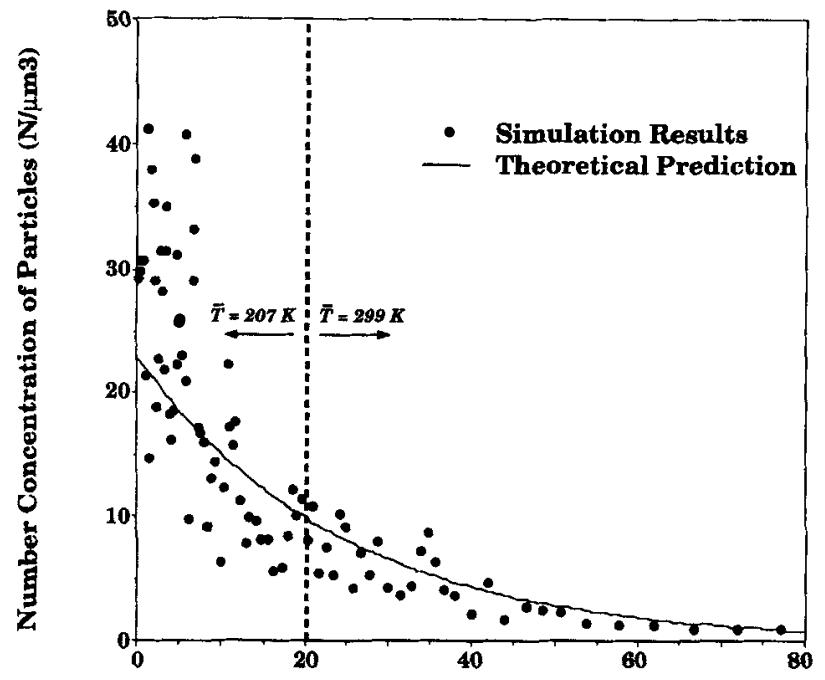

Height Above Container Bottom $(\mu \mathrm{m})$

Figure 6. Simulated and theoretical concentration profiles.

The particle $(0.119 \mu \mathrm{m}$ gold, $\rho=19.3)$ concentration profile at $298^{\circ} \mathrm{C}$ is determined using $5 \%$ of the particles in the center of the bed as a reference height and concentration. The results show the excellent agreement between the simulation data and the analytical solution.

Equation 11 predicts the relative concentration profile of the particles (i.e., the concentration at any $x$-position relative to any other $x$-position). For a reference point, we have chosen the average concentration at the center $x$-position in the simulated concentration data and have generated the theoretical concentration profile of this system (Figure 6). The theoretical curve shows good agreement with the concentration data obtained from the simulation, but, as expected, tends to deviate at heights close to the container bottom due to interactions with that solid boundary.

As an additional check on our simulation data, we have calculated the apparent temperature at specific heights from the container bottom in this system. By taking the concentrations at two $x$-positions in the simulation and inserting these values into Eq. 11, we can solve for the individual tempera; tures at specific points. If we average these temperatures both near and far away from the bottom, we see the expected cooling effect near the bottom of the container: $207 \mathrm{~K}$ for the particles below $20 \mu \mathrm{m}$ vs. $299 \mathrm{~K}$ for the particles above 20 $\mu \mathrm{m}$. This "cooling" is also seen as a disproportionately large concentration of particles near the bottom of the container (Figure 6).

The concentration profiles that form when colloidal particles are allowed to settle in a stationary fluid tend to become steeper as the diameter and/or density of the particles increases. At large diameters or high densities, the particles will no longer remain suspended and will form a packed bed of particles. Knowing this packed bed void fraction is necessary for a variety of applications, such as for calculating the overall system void fraction from the packed and expanded bed heights (Eq. 7). The packed bed void fraction can be found by interpolation between special cases of known packing fraction (Davis and Carter, 1990) or by a variety of experimental techniques.
Table 1. Simulated Void Fraction after a Specified Number of Moves and Decreasing Step Sizes

\begin{tabular}{clc}
\hline No. of Moves & Step Size & Void Fraction \\
\hline $1 \times 10^{7}$ & $0.1 \mathrm{D}$ & $0.459 \pm 0.013$ \\
$1 \times 10^{7}$ & $0.0066 \mathrm{D}$ & $0.446 \pm 0.003$ \\
$1 \times 10^{7}$ & $\mathbf{0 . 0 0 2 0 \mathrm { D }}$ & $0.437 \pm 0.001$ \\
$1 \times 10^{7}$ & $\mathbf{0 . 0 0 1 0 \mathrm { D }}$ & $0.4368 \pm 0.0008$ \\
\hline
\end{tabular}

We have used our simulation to calculate this packed bed void volume. One thousand particles were allowed to settle for $4 \times 10^{7}$ attempted moves while stepping at successively decreasing step sizes (Table 1). First, $10^{7}$ moves were attempted, then the average distance of the five nearest neighboring particles was calculated. The next step size was taken to be the distance of the second nearest neighboring particle to facilitate a rapid approach to a packed configuration. After four iterations of this procedure, a value for the packed bed void of $43.7 \%$ was calculated. This value is high but in the range of reported values of 0.35 to 0.44 for random packing (Bernal, 1959; Bernal and Mason, 1960; McGeary, 1961; Williams, 1986; Davis and Carter, 1990).

\section{Fluidized beds}

Steady-State Expansion. The same technique used to calculate colloid particle locations can be used to calculate fluidized bed expansion behavior. The physical motion of particles in fluidized beds, however, is not directly analogous to molecular motion. In particular, by using the product of Boltzmann's constant and system temperature to represent the average kinetic energy of the particles, we only account for random Brownian motion and not motion due to fluctuations in the fluid flow field. Since Brownian motion should be insignificant in fluidized beds, the simulation algorithm reduces to an energy minimization routine. However, the simulation still accurately predicts liquid fluidized bed expansion data. Modifications of the kinetic energy term can be made to attempt to incorporate fluid-induced fluctuations and will be discussed in the following section.

Figure 7a shows the expansion of uniform-sized stainlesssteel spheres fluidized with mineral oil. As one can see from the figure, the simulation results are nearly identical to Happel's model, and very closely approximate other recent models for predicting the expansion of fluidized beds (Foscolo et al., 1983; Richardson and Zaki, 1954; Hirata and Bulos, 1990). This excellent agreement between Happel's model (the model used in the simulation) and the simulation results suggests that the technique used in this work does not introduce any artifacts or numerical errors when simulating fluidized beds. The agreement also implies that more complicated fluidized systems, such as those with interparticle electrostatic or magnetic forces, should be able to be modeled with this type of simulation provided the appropriate equations governing the physical phenomena are included.

We have also attempted to characterize the expansion behavior of nonuniform (narrowly distributed) nickel and glass particles using the particle distribution's Sauter mean diameter. The expansion curves for both the nickel (Figure $7 \mathrm{~b}$ ) and glass (Figure 7c) exactly match those predicted by Happel's model, and follow the experimental expansion behavior of the particles quite well. Note that the force term used in this 


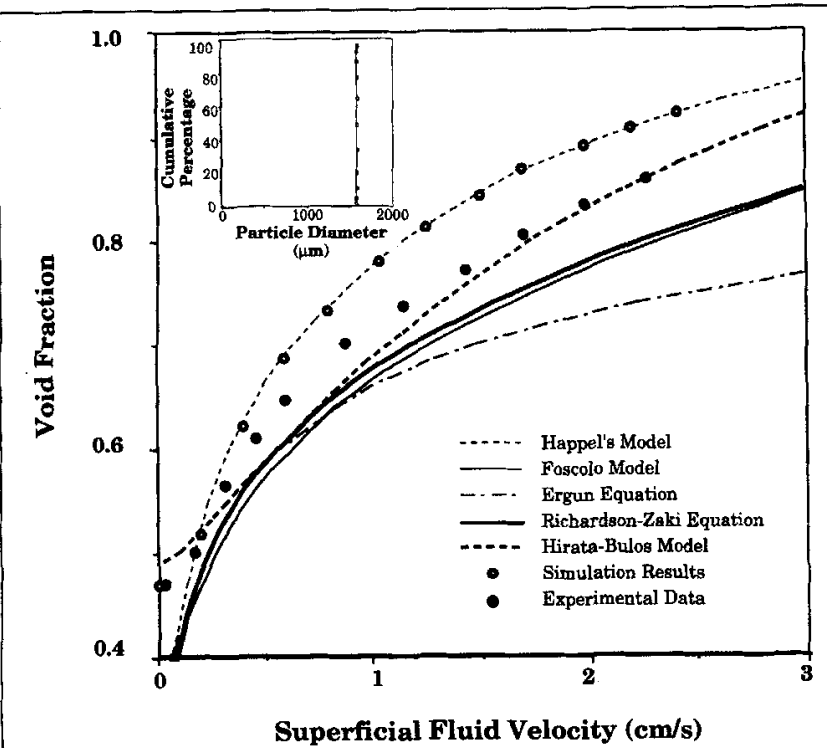

(a)

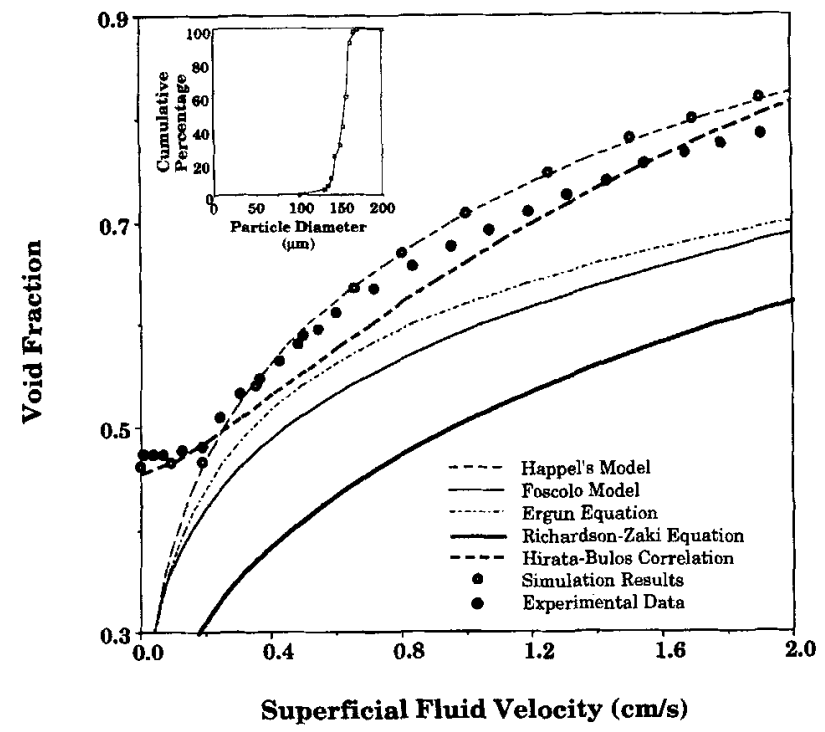

(b)

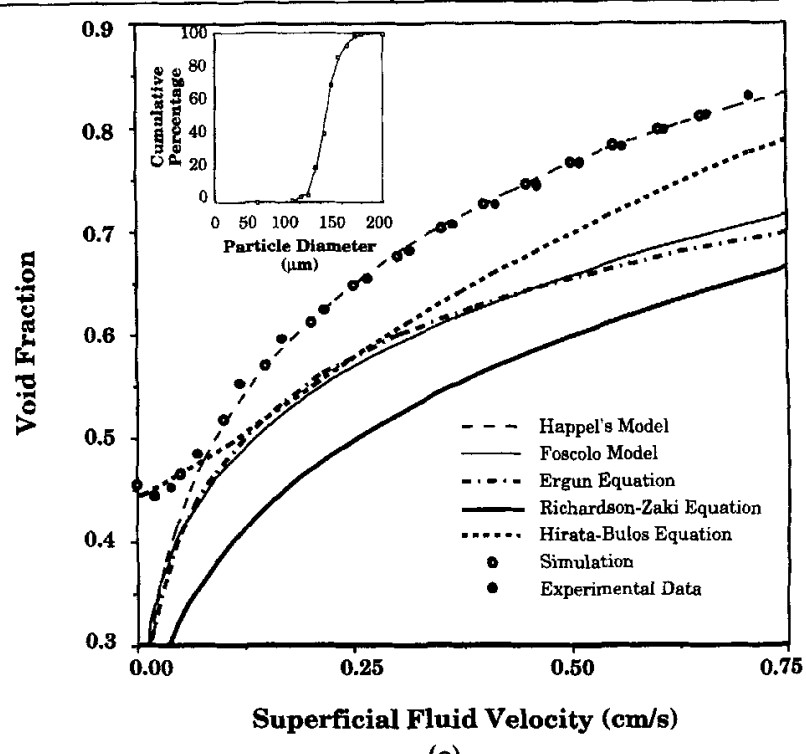

(c)

\section{Figure 7. Expansion curve for particle fluidization in} $25^{\circ} \mathrm{C}$ water and mineral oil.

Plot shows expansion characteristic of (a) $1588-\mu \mathrm{m}$ chrome steel bearings in mineral oil; (b) narrowly distributed nickel spheres; and (c) high-index glass spheres in $25^{\circ} \mathrm{C}$ water. The simulations exactly match Happel's model and agree well with the experimental data and the other correlations available for the prediction of bed voidage. derived for uniform spheres and, therefore, cannot accurately be used for nonuniform spheres. Other researchers have done alternative derivations of the viscous forces to include effects from a particle size distribution, and these results could be incorporated in this type of particle simulation (Tien, 1989).

Unsteady-State Expansion. The nature of this simulation allows us to simulate the more complicated dynamic behavior of a fluidized bed that cannot be derived from simple predictive models and mathematical correlations. To simulate dynamic behavior, we must derive an expression for the progressed time associated with a particular number of simulation moves. To calculate this progressed time, we have simulated a system of particles fluidized beyond their terminal velocity. The theoretical time required to move a given distance, $H$, is

$$
t=\frac{H}{U_{V}},
$$

where $U_{V}$ is the mean velocity of the particles in the direction of movement. For this situation, $U_{V}$ is simply the difference between the interstitial velocity of the surrounding fluid and the terminal velocity of the particles.

By operating this simulation for a fixed number of attempted steps, we can predict the time required for the particles to travel a fixed distance. The resulting equation for the time progressed in a fixed number of accepted moves is therefore given by

$$
t=\frac{M_{a c c} S}{2 N_{p} U_{V}},
$$

where $M_{a c c}$ is the number of accepted moves, $N_{p}$ is the number of particles in the representative system, and $S$ is the radius of the sphere formed by the locus of positions available for particle movement. Because downward moves are energetically unfavorable, the locus of positions available to 


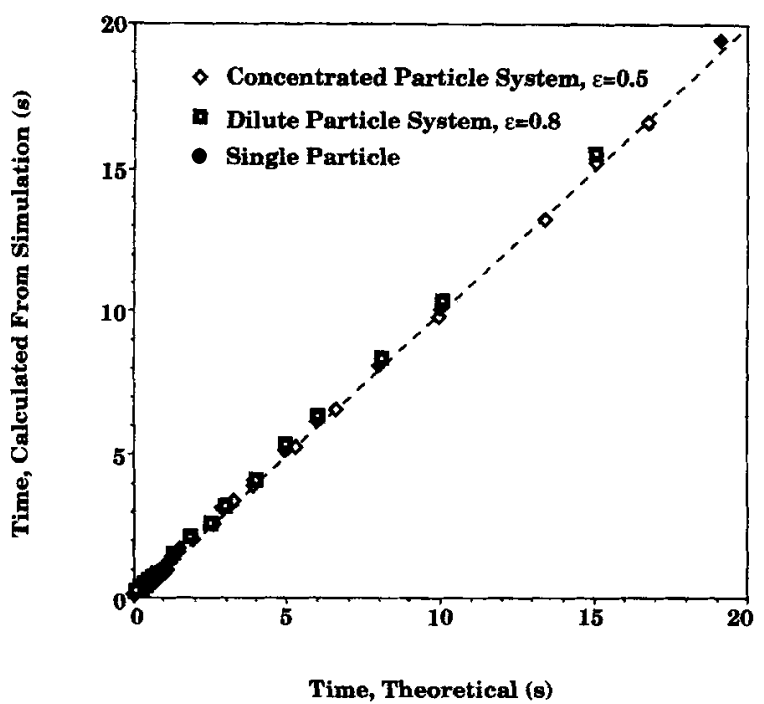

Figure 8. Simulation time vs. theoretical time.

\begin{abstract}
A system of particles $\left(d=150 \mu \mathrm{m}, \rho=8.91 \mathrm{~g} / \mathrm{cm}^{3}\right)$ fluidized beyond their terminal velocity $\left(u_{s}=300 \mathrm{~cm} / \mathrm{s}\right)$ is used to compare the theoretical time and the time calculated from the simulation. The plot shows excellent agreement between the two methods.
\end{abstract}

particle movement forms a hemispherical shell of radius $S$ above the selected particle's center. A plot of the calculated time using Eq. 13 vs. the theoretical time (Eq. 12) required to move the equivalent distance shows nearly exact agreement (Figure 8).

Although Eq. 13 can be used to convert the number of simulation moves into real time, the behavior observed is not independent of step size. By comparing data from dynamic expansion experiments to results from simulations with various step sizes, we can determine the step size that best repre-

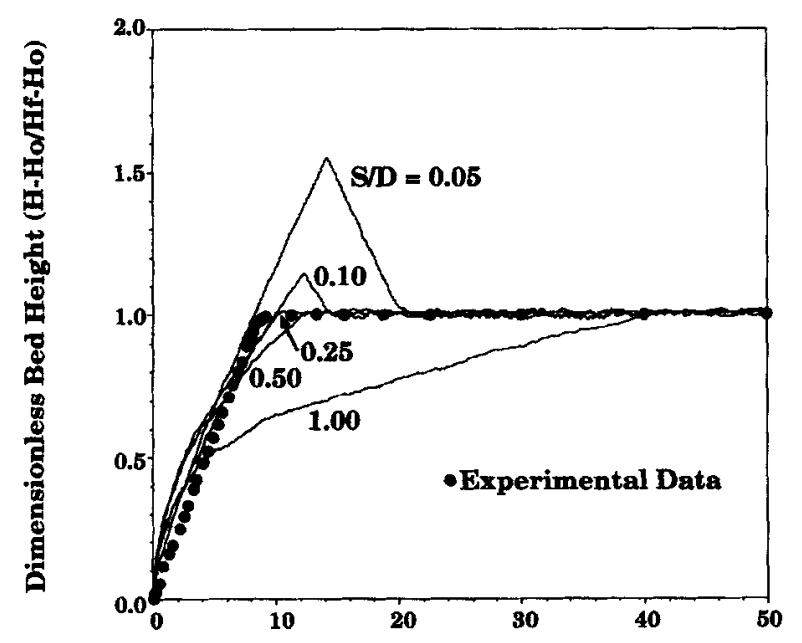

Time (s)

Figure 9. Dimensionless bed height as a function of time.

Results for experimental data and simulated results of various step sizes for stainless-steel spheres $\left(d_{p}=0.1588 \pm\right.$ $\left.0.0003 \mathrm{~cm}, \rho=7.63 \mathrm{~g} / \mathrm{cm}^{3}, U_{\mathrm{s}}=1.15 \mathrm{~cm} / \mathrm{s}\right)$. Too large and too small a step size result in significant overestimations of the time to reach steady-state bed height.

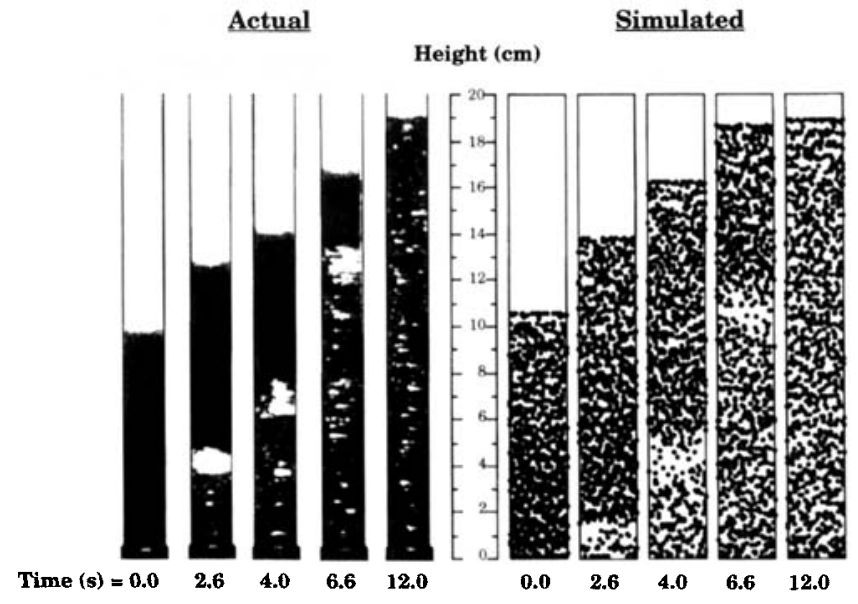

Figure 10. Actual and simulated expansion of particles in a fluidized bed.

As particles expand a plug of particles forms and particles drop away from the bottom of this plug to their steady-state void fraction. The simulated results match experimental observations of this phenomenon.

sents the dynamic behavior of the particles of interest. Figure 9 shows the results for the simulated expansion of stainlesssteel particles. By operating at $S / d_{p}$ between 0.1 and 0.25 , we obtain the best estimate for the dynamic approach of a fluidized bed to a fully expanded state. Note that these two bounds on the step size correspond to the average distance between nearest neighbor surfaces that would occur in a fluidized bed with voidages ranging from 0.55 to 0.70 (exactly the range of voidages shown in Figure 9).

In addition to macroscopic expansion behavior, the simulation can be used to predict dynamic microscopic structural phenomena. When fluid is introduced into a fluidized bed, particles begin to expand as a plug, maintaining their packed void fraction. Particles will fall away from the bottom surface of this plug to reach the steady state void fraction for that particular fluid velocity. Figure 10 shows how a time progression of an actual fluidized bed of stainless-steel spheres (1588 $\mu \mathrm{m}, \rho=7.63 \mathrm{~g} / \mathrm{cm}^{3}$ ) in mineral oil is nearly identical to the simulation results of the same system.

The particle arrangement in the settling of an expanded bed can also be predicted accurately by the simulation; particles form a plug at the bottom of the bed instead of at the top. However, accurate time predictions cannot be made without modifying the calculations. Figure 11 shows the experimental settling curve for stainless-steel spheres in mineral oil along with the simulated settling curves. The simulation results for four different step sizes all drastically overpredict the time for the system to settle if the interstitial fluid velocity is used in Eq. 13. This result is not surprising since the settling of the particles is governed by the terminal velocity of the particles and not the interstitial velocity. By replacing the interstitial velocity in Eq. 13 with the terminal velocity, we obtain a much closer representation of the dynamic behavior of these settling particles. Finally, if we include hindered settling using the average system void fraction (Geankoplis, 1983):

$$
U_{t}=\frac{g d_{p}^{2}\left(\rho_{p}-\rho_{f}\right)}{18 \mu}\left(\epsilon \psi_{p}\right)
$$




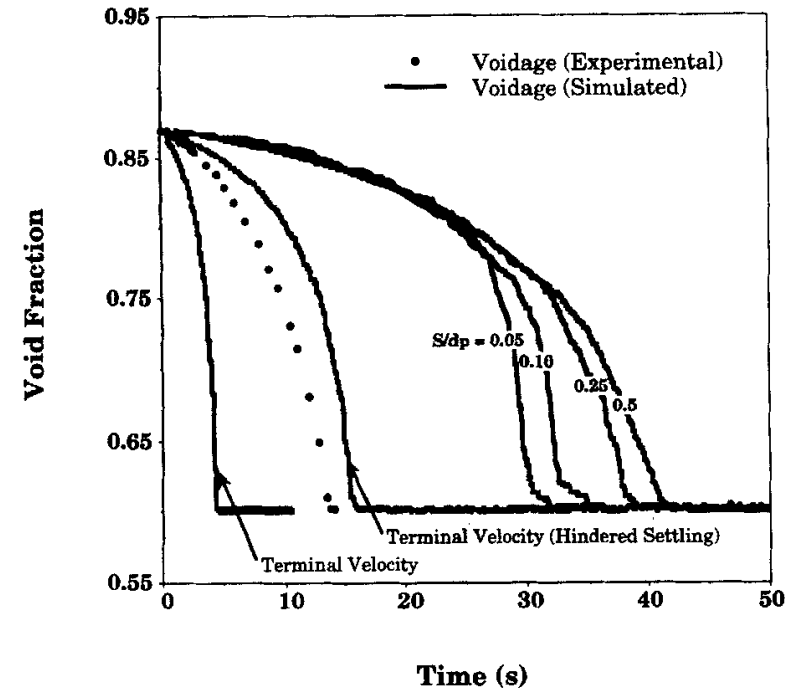

Figure 11. Simulation and experimental data for particles settling as a function of time.

Simulation results for stain]ess-steel spheres $\left(d_{p}=0.1588\right.$ $+0.0003 \mathrm{~cm}, \rho=7.63 \mathrm{~g} / \mathrm{cm}^{3}, U_{\mathrm{s}}=$ change from $2.0 \mathrm{~cm} / \mathrm{s}$ to $0.4 \mathrm{~cm} / \mathrm{s}$ ) settling at step sizes of $0.05 \mathrm{D}, 0.1 \mathrm{D}, 0.25 \mathrm{D}, 0.5$ $D$ exhibit the same general shape as the data, but overpre. dict the amount of time necessary to fully settle. Using the terminal velocity for the characteristic velocity $\left(S / d_{p}=0.1\right)$ yields a better representation of the time. Accounting for hindered settling (at the mean system void fraction) in the terminal velocity calculation results in better agreement between the simulation data and the experimental results.

where

$$
\psi_{p}=\frac{1}{10^{1.82(1-\epsilon)}},
$$

we find excellent agreement between the experimental data and the time predicted by the simulation.

While these results show that the simulation can predict the time behavior of fluidized beds, caution must be exercised in interpreting the results due to the limited physics that has been included in the model. For instance, consider a particle in the center of the rising plug in Figure 10. In the actual column, the particle is carried up the column by the particles below it. For the simulation, there are no particle-particle interactions; the particle in the simulation is moving up as fast as the particle above it will move out of the way. These behaviors, while slightly different phenomenologically, are still related to the fact that an effective pressure drop is being created across the plug, which in turn is causing the plug to move upward in the fluidized bed.

Kinetic Energy. Many additions can be made to the simulation to increase the physical accuracy of the predictions and expand the method's applicability. For instance, the kinetic energy used in the simulations was the product of the Boltzmann's constant and system temperature. Physically, this term includes the random Brownian motion of fluidized particles, but ignores the semirandom fluctuations of particles due to fluid motion. Although the fluid motion is not wholly random, we can approximate the effect of fluid-motion fluctuations on the expansion behavior of fluidized beds by using the velocity of the moving fluid in the definition of the parti-

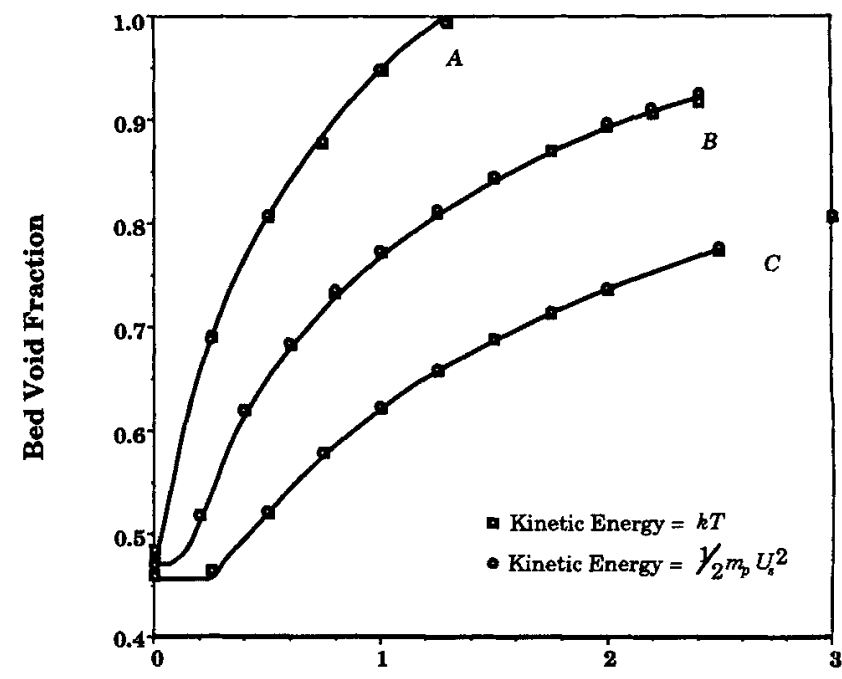

Superficial Fluid Velocity $(\mathrm{cm} / \mathrm{s})$

Figure 12. Simulation of the characteristic expansion curves for three different fluid-particle systems.

The threc systems, $A\left(R e_{p}=0.025, d_{p}=775 \mu \mathrm{m}, \rho_{p}=1.5\right.$ $\left.\mathrm{g} / \mathrm{cm}^{3}, \rho_{f}=1.0 \mathrm{~g} / \mathrm{cm}^{3}, \mu=1.0 \mathrm{cP}\right), B\left(R e_{p}=1.0, d_{p}=1588\right.$ $\left.\mu \mathrm{m}, \rho_{p}=7.6 \mathrm{~g} / \mathrm{cm}^{3}, \rho_{f}=0.869 \mathrm{~g} / \mathrm{cm}^{3}, \mu=139 \mathrm{cP}\right)$, and $C$ $\left(R e_{p}=2.5, d_{p}=500 \mu \mathrm{m}, \rho_{p}=2.2 \mathrm{~g} / \mathrm{cm}^{3}, \rho_{f}=2.0 \mathrm{~g} / \mathrm{cm}^{3}\right.$, $\mu=1.0 \mathrm{cP}$ ), are simulated using two methods of calculating the kinetic energy for acceptance probability. Using either the equation for kinetic energy of a macroscopic body $\left(1 / 2 m_{p} U_{s}^{2}\right)$ or the product of Boltzmann's constant and temperature $(k T)$ results in identical expansion curves.

cle's kinetic energy. The average kinetic energy of a fluidized particle can be taken to have the form

$$
K E=c m_{p} U_{I}^{2}
$$

where $m_{p}$ is the particle mass, $U_{I}$ is the interstitial velocity of the fluidizing media, and the constant $c$ is generally considered to have the value of $1 / 2$ or $1 / 3$ (Buyevich, 1994; Buyevich and Kapbasov, 1994; Koch, 1990; Nettleton, 1993).

We have replaced the kinetic energy term in the acceptance probability (Eq. 1) with Eq. 16 and, using $c=1 / 2$, have resimulated the expansion characteristics of the uniformly sized particles in liquid fluidized beds. The results, shown in Figure 12, are identical to those obtained with $K E=k T$. For the low Reynolds number flow of liquid-fluidized beds, this result is not surprising since the inertial forces are lower than the viscous forces. The insignificant contribution of the particles momentum can be seen mathematically by comparing the value of $K E$ from Eq. $16\left(0.06 \mathrm{~g} \cdot \mathrm{cm}^{2} / \mathrm{s}^{2}\right.$ for stainless steel fluidized at a superficial velocity of $2.5 \mathrm{~cm} / \mathrm{s}$ ) to the change in energy of a typical vertical move at identical conditions $\left(15.5 \mathrm{~g} \cdot \mathrm{cm}^{2} / \mathrm{s}^{2}\right)$.

For higher particle Reynolds numbers, such as those typically encountered in gas fluidized beds, the contribution of the redefined kinetic energy term is far from insignificant. To simulate these gas-fluidized beds, we have replaced Happel's model, which was specifically derived for low Reynolds systems, with the Ergun equation for the calculation of fluid forces. We chose the Ergun equation because it is suitable for turbulent systems and is designed for packed beds (Ergun, 


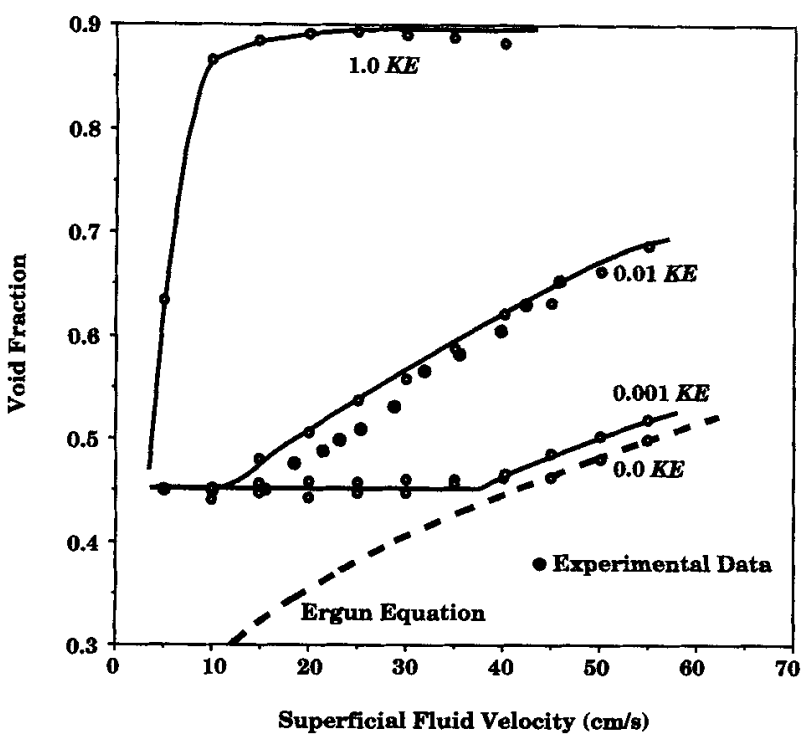

Figure 13. Experimental, simulated, and theoretical expansion curves for a gas fluidized bed.

Various forms of the kinetic energy result in different curves for the expansion characteristic of $90-\mu \mathrm{m}$ nickel particles. The theoretical expression signficantly underpredicts experimental results, while the simulation that utilizes the same theoretical expression overpredicts the expansion curve. Reducing the velocity used in the calculation of $K E$ by an order of magnitude $(0.01 K E)$ results in an accurate prediction of experimental results.

1952). Correlations for fluid forces in fluidized beds may lump the effects of particle motion into the calculation of fluid forces and were therefore not used.

We would expect that using Eq. 16 for the average kinetic energy of the particles would yield a larger simulated steady state bed height (void fraction) than that predicted by the Ergun equation. Figure 13 shows that this is indeed true; the "fluid fluctuations" expand the bed much like Brownian motion expands colloidal particles. The Ergun equation does not account for this expansion, and therefore underpredicts the experimental data. Unfortunately, the simulations overpredict the data, indicating that the equations found in the literature for the kinetic energy of fluidized particles may not be accurate. The overprediction is most likely due to the fact that the particles move relative to each other at velocities significantly lower than the interstitial fluid velocity. A linear reduction of the particle velocity in the kinetic energy term by an order of magnitude aligns the simulated results to the experimental expansion curves.

\section{Conclusions}

We have developed a discrete particle simulation, analogous to a statistical mechanical simulation for molecular systems, to describe particle behavior in colloidal suspensions and fluidized beds. This statistical-mechanical-type simulation describes the macroscopic behavior of fluid-particle systems using the microscopic forces that affect the movement of individual particles. We have shown this model to be useful in predicting packing fractions for static systems, concentration profiles in colloidal suspensions, and both static and dynamic expansion characteristics of fluidized beds.
This simulation is easily modified to include other physical parameters that may affect the final steady-state behavior of particle systems. Electrostatic, magnetic or frictional interparticle forces can be added to the simulation to calculate the effect of these forces on the expansion behavior. Alternative forms for the hydrodynamic model can also be easily incorporated into the force calculations, including correlativetype predictions and effects from adjacent particles (Durlofsky and Brady, 1987; Brady et al., 1988; Brady, 1988). Fluidized beds containing mixtures of particles (i.e., different sizes and/or densities) can also be studied. Such fluidized beds exhibit a variety of interesting spatial phenomena including classification, segregation, and inversion, all of which should be able to be predicted using this technique.

\section{Acknowledgments}

The authors would like to acknowledge the financial support of The National Science Foundation, the Donors of the Petroleum Research Fund (administered by the American Chemical Society), and the University of Michigan. We also wish to thank Dr. Robert Ziff for his help on this research.

\section{Notation}

$$
\begin{aligned}
E_{t} & =\text { total system energy } \\
L & =\text { length of packed bed of particles } \\
t & =\text { time } \\
U_{s} & =\text { superficial fluid velocity } \\
U_{t} & =\text { particle terminal velocity } \\
X & =\text { coordinate in storage of particle locations } \\
Y & =\text { coordinate in storage of particle locations } \\
Y_{\text {old }} & =\text { initial } Y \text { coordinate of a selected particle } \\
Y_{\text {new }} & =\text { newly generated } Y \text { coordinate of a selected particle } \\
Z & =\text { coordinate in storage of particle locations } \\
\Delta p & =\text { pressure drop in a packed bed }
\end{aligned}
$$

\section{Subscripts}

$$
\begin{aligned}
& b=\text { buoyancy } \\
& g=\text { gravity }
\end{aligned}
$$

\section{Literature Cited}

Barnea, E., and J. Mizrahi, "A Generalized Approach to the Fluid Dynamics of Particulate Systems: 1. General Correlation for Fluidization and Sedimentation in Solid Multiparticle Systems," Chem. Eng. J., 5, 171 (1973)

Bernal, J. D., "Geometry of the Structure of Monatomic Liquids," Nature, 185, 68 (1959).

Bernal, J. D., and J. Mason, "Co-ordination of Randomly Packed Spheres," Nature, 188, 910 (1960).

Brady, J. F., "Stokesian Dynamics," Ann. Rev. Fluid. Mech., 20, 111 (1988).

Brady, J. F., R. J. Phillips, J. C. Lester, and G. Bossis, "Dynamic Simulation of Hydrodynamically Interacting Suspensions," J. Fluid Mech., 195, 257 (1988).

Brinkman, H. C., "A Calculation of the Viscous Force Exerted by a Flowing Fluid on a Dense Swarm of Particles," Appl. Sci. Res., A1, 27 (1947).

Buyevich, Y. A., "Fluid Dynamics of Coarse Dispersions," Chem. Eng. Sci., 49, 1217 (1994).

Buyevich, Y. A., and S. K. Kapbasov, "Random Fluctuations in a Fluidized Bed," Chem. Eng. Sci., 49, 1229 (1994).

Ciccotti, G., D. Frenkel, and I. R. McDonald, Simulation of Liquids and Solids, Elsevier, New York (1987).

Davis, I. L., and R. G. Carter, "Random Particle Packing by Reduced Dimension Algorithms," J. Appl. Phys., 67, 1022 (1990).

Dickinson, E., The Structure, Dynamics and Equilibrium Properties of Colloidal Systems, D. M. Bloor and E. E. Wyn-Jones, Kluwer, Dordrect, The Netherlands (1990). 
Dickinson, E., and S. Euston, "Monte Carlo Simulation of Colloidal Systems," Adv. Colloid Interface Sci., 42, 89 (1992).

Durlofsky, L., and J. F. Brady, "Dynamic Simulation of Hydrodynamically Interacting Particles," J. Fluid Mech., 180, 21 (1987).

Ergun, S., "Fluid Flow Through Packed Columns," Chem. Eng. Prog., 48, 89 (1952).

Fleer, G. J., J. M. H. Scheutjens, and M. A. Cohen-Stuart, Colloids and Surf., 31, (1988).

Fleer, G. J., and J. Lyklema, Adsorption from Solution at the Solid/Liquid Interface, Academic Press, New York (1983).

Foscolo, P. U., L. G. Gibilaro, and S. P. Waldram, "A Unified Model for Particulate Expansion of Fluidised Beds and Flow in Fixed Porous Media," Chem. Eng. Sci., 38, 1251 (1983).

Garside, J., and M. R. Al-Dibouni, "Velocity-Voidage Relationships for Fluidization and Sedimentation in Solid-Liquid Systems," Ind. Eng. Chem., Process Des. Dev., 16, 206 (1977).

Geankoplis, C. J., Transport Processes and Unit Operations, PrenticeHall, Englewood Cliffs, NJ (1983).

Gubbins, K. E., and A. Z. Panagiotopoulos, "Molecular Simulation," Chem. Eng. Prog., 10, 23 (1989).

Happel, J., "Viscous Flow in Multiparticle Systems: Slow Motion of Fluids Relative to Beds of Spherical Particles," AIChE J., 4, 197 (1958).

Hiemenz, P. C., Principles of Colloid and Surface Chemistry, Dekker, New York (1986).

Hirata, A., and F. B. Bulos, "Predicting Bed Voidage in Solid-Liquid Fluidization," J. Chem. Eng. Jap., 23, 599 (1990).

Hirtzel, C. S., and R. Rajagopolan, Colloidal Phenomena: Aduanced Topics, Noyes, Park Ridge, NJ (1985)

Hutchinson, H. R., and D. N. Sutherland, "An Open Structure Random Solid," Nature, 206, 1036 (1965).

Jean, R., and L. Fan, "A Fluid Mechanic-Based Model for Sedimentation and Fluidization at Low Reynolds Numbers," Chem. Eng. Sci, 44, 353 (1989).

Jottrand, R., "An Experimental Study of the Mechanism of Fluidization," J. Appl. Chem., 2, Suppl. Issue, 1, 517 (1952).

Koch, D. L., "Kinetic Theory for a Monodisperse Gas-Solid Suspension," Phys. Fluids A, 10, 1711 (1990).

Kuwabara, S., "The Forces Experienced by Randomly Distributed Parallel Circular Cylinders or Spheres in a Viscous Flow at Small Reynolds Numbers," J. Phys. Soc. Jap., 14, 527 (1959).

Letan, R., "On Vertical Dispersed Two-Phase Flow," Chem. Eng. Sci., 29, 621 (1974).

Lewis, E. W., and E. W. Bowerman, "Fluidization of Solid Particles in Liquids," Chem. Eng. Prog., 48, 603 (1952).
Lewis, W. K., E. R. Gilliland, and W. C. Bauer, "Characteristics of Fluidized Particles," Ind. Eng. Chem., 41, 1104 (1949).

Loeffler, A. L., and B. F. Ruth, "Particulate Fluidization and Sedimentation of Spheres," AIChE J., 5, 310 (1959).

McGeary, R. K., "Mechanical Packing of Spherical Particles," J. Am. Ceram. Soc., 44, 513 (1961).

Nettleton, R. E., "On the Relation between Thermodynamic Temperature and Kinetic Energy per Particle," Can. J. Phys., 72, 106 (1993).

Oliver, D. R., "The Sedimentation of Suspensions of Closely-Sized Spherical Particles," Chem. Eng. Sci., 15, 230 (1961).

Rapagna, S., R. Di Felice, L. G. Gibilaro, and P. U. Foscolo, "Steady-state Expansion Characteristics of Monosize Spheres Fluidised by Liquids," Chem. Eng. Commun., 79, 131 (1989).

Richardson, J. F., and W. N. Zaki, "Sedimentation and Fluidisation: I," Trans. Inst. Chem. Eng., 32, 35 (1954).

Richardson, J. F., and W. N. Zaki, "The Sedimentation of a Suspension of Uniform Spheres Under Conditions of Viscous Flow," Chem. Eng. Sci, 3, 65 (1954).

Snook, I., and W. van Megen, "Colloidal Properties from First Principles," Chem. Phys. Lett., 33(1), 156 (1975).

Steinour, H. H., "Nonflocculated Suspensions of Uniform Spheres," Ind. Eng. Chem., 36, 618 (1944).

Sutherland, D. N., "Comment on Vold's Simulation of Floc Formation," J. Colloid Interface Sci., 22, 300 (1966).

Takahashi, A., and M. Kawaguchi, "The Structure of Macromolecules Adsorbed on Interfaces," Adv. Poly. Sci., 46, 1 (1982).

Tien, Chi., Granular Filtration of Aerosols and Hydrosols, Butterworths, Stoneham, MA (1989).

Vincent, B., and S. B. Whittington, Surface and Colloid Science, Vol. 12, Plenum Press, New York (1982)

Vold, M. J., "Computer Simulation of Floc Formation in a Colloidal Suspension," J. Colloid Interf. Sci., 18, 684 (1963).

Wen, C. Y., and L. S. Fan, "Some Remarks on the Correlation of Bed Expansion in Liquid-Solid Fluidized Beds," Ind. Eng. Chem., Process Des. Dev., 13, 194 (1974).

Wen, C. Y., and Y. Ho Yu, "Mechanics of Fluidization," AlChE Symp. Ser., 62, 100 (1966).

Williams, D. E. G., "Packing Fractions in Random Close Packing," $J$. Non-Cryst. Solids, 86, 1 (1986).

Manuscript received Oct. 17, 1994, and revision received May 9, 1995 